\title{
Naval Diving Training Management Model Analyzed by Safety Risk Assessment
}

\author{
Hu Pei \\ Navy Submarine Academy \\ Qingdao, China \\ qdhupei@163.com
}

\author{
Hao Qian \\ Qingdao Campus of Navy Aeronautical University \\ Qingdao, China
}

\author{
Han Nana \\ Qingdao Campus of Navy Aeronautical University \\ Qingdao, China
}

\begin{abstract}
Our object in this report is to analyze and assess risks in diving train, and ensure fresh divers' safety underwater. We develop a diving training management safety assessment (DTMSA) model which is based on the safe risk matrix. We can identify and evaluate all influencing factors in diving train, and confirm the risk rating according to DTMSA standards. In actual training, it is a simple and effective safety risk control tool which is used to investigate problems in operation and management so as to sum up and deal with it in time. We suggest to start a further research in all diving process and unit, in order to establish a whole new diving safety risk assessment system for Navy.
\end{abstract}

\section{Keywords—Diving; Safety; Risk; Assessment; Matrix}

\section{INTRODUCTION}

September 6, 2017, two divers explore the Great Wall together underwater, but both be missing in Panjiakou reservoir, Hebei province. After 12 days, their bodies had been found by underwater machine. According to published reports, they are top qualified divers, also can be support 6-8 hours diving activities under water in the sufficient equipment, but this activity was only passed 2 hours and met accident.

The accident once again to let all public know a very serious topic-the dive is dangerous. As the diving instructors in the Navy diving train center, we always face a problem is that most of our students are diving "rookie". For protect their safety in training, we have been actively exploring solutions some foreign companies and professional diving training institutions which early use the risk assessment to control and supervise their diving team and diving, and achieved good results. We learn these advanced diving organization management and training experience, combined with our unique diving training procedure, developed the potential Naval Diving Train Management model based on the safety risk assessment.

\section{RISK ASSESSMENT THEORY}

\section{A. RISK ASSESSMENT}

Risk assessment is the determination of quantitative or qualitative estimate of risk related to a well-defined situation and a recognized threat (also called hazard). Quantitative risk assessment requires calculations of two components of risk (R) the potential consequences of an adverse event(c), and the probability (p) that the consequences will occur. An acceptable risk is a risk that is understood and tolerated usually because the cost or difficulty of implementing an effective countermeasure for the associated vulnerability exceeds the expectation of consequences.

In the engineering of complex systems, sophisticated risk assessments are often made within safety engineering and reliability engineering when it concerns threats to life, environment or machine functioning. The agriculture, nuclear, aerospace, oil, rail and military industries have a long history of dealing with risk assessment. Also, medical, hospital, social service and food industries control risks and perform risk assessments on a continual basis. Methods for assessment of risk may differ between industries and whether it pertains to general financial decisions or environmental, ecological, or public health risk assessment [4].

Professor Du Duanfu believes that risk refers to the uncertainty of loss. It is the synthesis of multiple negative deviations caused by the uncertainty of the decisions and objective conditions of future actions, and be given the following mathematical formulas [3]:

$$
R=f(P, C)
$$

Equation (1):

$$
\begin{aligned}
& \mathrm{R} \text {-risk; } \\
& \mathrm{P} \text {-The probability of an adverse event; }
\end{aligned}
$$


C -The consequences of an adverse event.

The definition of this risk proposed by Professor Du Duanfu has now been widely used.

When the income is difficult to quantify, or when compared with the loss, the benefit is negligible, the game of income and loss will be transformed into a game between various potential losses (The safety risk is this type of typical problem).So Equation (1) can continue to be simplified as a form of multiplication as a specific form of function:

$$
\mathrm{R}=\mathrm{p} \times \mathrm{c}
$$

\section{B. RISK MATRIX}

A Risk matrix is a matrix that is used during risk assessment to define the level of risk by considering the category of probability or likelihood against the category of consequence severity. This is a simple mechanism to increase visibility of risks and assist management decision making [1] [5].

This method is put forward to buy Engineering Team in the United States Air Force Electronic Systems Center in April 1995. Since 1996, many projects are taken to evaluate project risk by risk matrix method [2].

Quantitative risk analysis depends on the impact of risk and risk probability of these two parameters. Measurement of risk affect the project once the risk degree is refers to the possible effect on the project caused by the size of the risk probability is the percentage; possibility of risk that is usually a subjective probability.

Equation (2) tells us, if we would get two key factors, one is the probability of an adverse event (P), and another is the consequences of an adverse event (C). In the risk matrix, we use another word "severity" to replace the factor word "consequences".

So, in the risk matrix, the harm severity can be categorized as 'Catastrophic', 'Critical', 'Marginal' and 'Negligible'.

The probability of harm occurring might be categorized as 'Certain', 'Likely', 'Possible', 'Unlikely' and 'Rare'. However it must be considered that very low probabilities may not be very reliable.

The company or organization then would calculate what levels of risk they can take with different events. This would be done by weighing up the risk of an event occurring against the cost to implement safety and the benefit gained from it.

\section{Diving Train Management SAFETy Assessment}

For Navy diving training institutions, we have been using all kinds of safety risk assessment methods to manage and control various diving operations in recent years. Based on risk matrix, diving training management safety assessment (DTMSA) model is such a successful and satisfactory attempt.
The DTMSA is a comprehensive inspection program designed to assess the operational readiness and adherence to existing regulations, requirements, policies, and standards of Naval diving units and personnel. The major areas evaluated during an assessment include: administration, training, diving equipment and storage, divers compressor(s) and system components, support equipment, diving and rescue procedures. These assessments will be conducted by a DTMSA Team (DT) consisting of Naval Diving Safety Officer (NDSO) and/or Naval Diving Train Center (NDTC) representatives serving as DTMSA Inspectors (DI) and/or Diving Medical Officer (DMO).

DTMSA is a risk control tool which investigates problems at an operational and administrative level so they may be viewed and addressed. The criteria for selecting facilities to undergo compliance assessment must be attuned to the different levels and types of diving activity performed by a unit and address the controls needed to eliminate deficiencies at their root causes. The purpose of the DTMSA program is to preserve Naval Diving resources by identifying gaps in compliance with Safety and Occupational Health Regulations.

\section{A. Probability and Severity Rating}

Risk is usually rated according to the seriousness of an accident and the probability of the accident taking place. For example, a destroy crash to sink is a serious and usually fatal accident but is very unlikely. Thus flying has a low hazard rating. In a military cross-country training minor injuries are quite possible and this has a medium hazard rating.

The risk matrix is a simpler safety assessment method, which is adequate for physical risk management, evaluate only the probability and the seriousness of the accident. More sophisticated methods, which may be carried out using specially designed software, also include the frequency of exposure to the hazard and consider financial as well as physical risk.

The probability of a diving accident occurring can be rated on a scale of A to E by DTMSA:

TABLE I. PROBABILITY RATING

\begin{tabular}{|c|c|c|}
\hline Label & Probability & Introduction \\
\hline A & Frequent & Occurs often in career/equipment service life. \\
\hline B & Likely & Occurs several times in career/equipment life. \\
\hline C & Occasional & Occurs sometime in career/equipment life. \\
\hline D & Seldom & Possible to occur in career/equipment life. \\
\hline E & Unlikely & Can assume will rarely occur in career/equipment \\
life.
\end{tabular}

The severity of an accident can be rated in a similar way: 
TABLE II. SEVERITY RATING

\begin{tabular}{|c|c|c|}
\hline Label & Severity & Introduction \\
\hline I & Catastrophic & $\begin{array}{c}\text { Complete mission failure, death, or loss of a diver's } \\
\text { air system. }\end{array}$ \\
\hline II & Critical & $\begin{array}{c}\text { Chief mission degradation, severe injury, } \\
\text { occupational illness, or critical system damage. }\end{array}$ \\
\hline III & Major & $\begin{array}{c}\text { Key mission degradation, injury, minor } \\
\text { occupational illness, or major system damage. }\end{array}$ \\
\hline IV & Minor & $\begin{array}{c}\text { Trivial mission degradation, injury, occupational } \\
\text { illness, or minor system damage. }\end{array}$ \\
\hline V & Negligible & $\begin{array}{c}\text { Minimal mission degradation, injury, occupational } \\
\text { illness, or negligible system damage. }\end{array}$ \\
\hline
\end{tabular}

\section{B. DTMSA Risk Rating Code}

DUSA findings have the potential to affect the health and safety of the individual diver and the safety of the facility or equipment used by divers to conduct operations. By utilizing a 'probability/severity' Risk Assessment Code (RAC) matrix, a RAC value is assigned to each finding. The final rating of the unit's inspection is based on the lowest RAC assigned which may affect the unit's authorization to dive. Finding Ratings are described below:

- RAC 1 - Unit is suspended from conducting diving operations until reauthorized by the NDSO. Indicates substantial probability that death or serious physical harm could result, and management knew or should have known of the hazard. The DT has the authority to immediately halt diving operations that in their opinion are unsafe. The findings are immediately confirmed with the NDSO.Ex: Flagrant lack of compliance with Naval regulations, standards, and procedures. Inadequate diver retrieval, support equipment etc.

- RAC 2 - A temporary suspension is issued until all Corrective Actions (CA) are completed, prior to the authorization to continue diving operations is granted by the Line Office Diving Officer (LODO).Indicates moderate probability injury or illness. At least one area evaluated does not meet the minimum requirements for safe diving operations or the unit is unable to demonstrate or maintain the minimum equipment, personnel, or level of dive proficiency in order to comply with Naval diving standards. A temporary suspension is issued until CA are completed prior to the authorization to continue diving operations by the LODO.Ex: Dive unit is non-compliant with maintaining appropriate life-saving equipment or personnel to conduct diving operations.

- $\mathrm{RAC} 3$ - It is required that CA are completed and confirmed by LODO within 30 days of the issued CA Plan. Indicates that equipment or divers must be removed from operations due to lack of appropriate maintenance or authorization. The potential for injury to the diver or injury to others if the equipment or diver is utilized in an unauthorized status prior to completion of CA.Ex: Critical equipment is out of service, has not been properly removed from service or is in need of immediate repair.
- RAC 4 - Complete CA Plan confirmed by LODO.Suggests programmatic, written, or other administrative deficiencies with minor probability of resulting in an injury or illness.Ex: Unit is unaware of proper forms and administrative procedures to follow.

- RAC 5 - Complete CA Plan confirmed by LODO. Majority of areas evaluated meets the minimum requirements. Any recommended CA will not interfere with safe diving operations.Ex: Minor administrative issues.

\section{Risk Rating}

On the basis of the probability and severity ratings, a risk rating matrix can be constructed to give DTMSA Risk Rating Code.

TABLE III. RISK RATING

\begin{tabular}{|c|c|c|c|c|c|}
\hline \multirow{2}{*}{ Probability } & \multicolumn{5}{|c|}{ Severity } \\
\cline { 2 - 6 } & $I$ & $I I$ & II & $V$ & $V I$ \\
\hline $\boldsymbol{A}$ & 1 & 1 & 1 & 2 & 2 \\
\hline $\boldsymbol{B}$ & 1 & 1 & 2 & 3 & 3 \\
\hline $\boldsymbol{C}$ & 1 & 2 & 3 & 4 & 4 \\
\hline $\boldsymbol{D}$ & 2 & 3 & 4 & 5 & 5 \\
\hline $\boldsymbol{E}$ & 2 & 3 & 4 & 5 & 5 \\
\hline
\end{tabular}

\section{Actual Application}

According to the DTSAM project need, we picked up some diving and underwater warfare experts to prepare the expert committee. In order to avoid the error assessment, the number of experts are more than 10 people. Experts need in-depth research and analysis from various aspects of diving training, combined with their own experience and theoretical knowledge to identify risk indicators, and to evaluate the index.

We also collect a large number of diving accidents data to improve the risk assessment model formula.

Ex: The British Sub Aqua Club reported 364 diving incidents in 2010, including 98 decompression incidents and 17 fatalities [6].

Ex: The Health and Safety Executive reported 24 fatal accidents in the 8-year period from 1996/97 to 2003/04, many amongst people receiving recreational diving training by instructors [6].

Ex: We analyze the cause of death in 1989-2009, and get these information. In all cause of death case [6]:

Cardiac Arrest, 35 person, 31.5\%;

Unknown, 31person, 27.9\%;

Drowning, 30person, 27.0\%; 
Embolism, 13person, 11.7\%;

Asphyxiation, 1person, $0.9 \%$;

Asthma, 1person, $0.9 \%$.

So, from these data, the DTSAM expert committee analyzed the risk factors which affect the diving safety, and determined the risk index is. These risk indicators can be applied to the risk matrix to indicate the risk level of an action.

General risk: diving plan, diving program, etc;

Diver health risk: age, health condition, physical condition, technical level, etc.
Diving equipment risk: new/old level, proficiency, maintenance, damage, etc;

Training platform risk: training site, training ship, operation platform, etc.

Hydrometeorological risk: training sea area, season, time, etc;

Emergency risk: Diver lost, Comms Loss, decompression sickness, Mechanical injury, etc.

TABLE IV. RISK RATING IN ACTUAL DIVING TRAIN

\begin{tabular}{|c|c|c|c|c|c|c|}
\hline Risk & Probabily & Effect & $\begin{array}{c}\text { Risk } \\
\text { Level }\end{array}$ & $\begin{array}{c}\text { Residual } \\
\text { Probability }\end{array}$ & $\begin{array}{c}\text { Residual } \\
\text { Effect }\end{array}$ & $\begin{array}{c}\text { Residual } \\
\text { Risk }\end{array}$ \\
\hline General & NA & NA & NA & NA & NA & NA \\
\hline Lost diver & $\mathrm{C}$ & $\mathrm{I}$ & 2 & $\mathrm{D}$ & $\mathrm{I}$ & 3 \\
\hline Unconscious diver & $\mathrm{C}$ & I & 2 & $\mathrm{D}$ & I & 3 \\
\hline AGE & $\mathrm{C}$ & I & 2 & $\mathrm{D}$ & II & 4 \\
\hline DCS & $\mathrm{C}$ & II & 3 & NA & NA & NA \\
\hline $\begin{array}{c}\text { Rig } \\
\text { Malfunction }\end{array}$ & B & III & 3 & $\mathrm{D}$ & I & 3 \\
\hline Mechanical Injury & $\mathrm{C}$ & II & 3 & $\mathrm{D}$ & I & 3 \\
\hline Loss of Comms & $\mathrm{B}$ & IV & 4 & $\mathrm{D}$ & II & 4 \\
\hline $\begin{array}{l}\text { Small boat } \\
\text { malfunction }\end{array}$ & B & II & 2 & NA & NA & NA \\
\hline
\end{tabular}

In actual diving train, for example, If, a chamber transfer lock door were left open, an accident might occur if the trunking door were also left open and an attempt was made to remove the trunking clamp under pressure. The chances of this happening are small and the probability rating would be 2 . If such an accident did occur, however, it would result in fatalities, giving a severity rating of 5. From the matrix, leaving the transfer lock door open has a medium hazard rating.

There may be other risks associated with leaving the door open. An unsecured door could swing in a heavy sea causing injury or damage to valves or fittings. In some operations there may also be a risk associated with gases from a contaminated bell atmosphere.

The risk assessment should also consider the risks of keeping the door closed. What, for example, are the risks associated with the divers being trapped in their chamber if the door seals?

\section{CONCLUSION}

It is not necessary to carry out risk assessment to a very detailed level and list every trivial hazard. Common sense will usually show how far to go.

Almost all risks are avoidable and frequently caused by a failure to follow procedures. Even equipment failure can usually be attributed to incorrect use or inadequate maintenance.
When Naval diving units use regular safety risk assessment method or toolbox to raise safety issues and allow feedback from the team. Other personnel who are not members of the diving team may also be involved in the diving operation. They should be briefed and allowances must be made for their lack of diving and safety risk assessment knowledge.

Every member of the DTMSA team must follow the procedures laid down and should also understand why they are following them. After a period of time, when the team has become completely familiar with procedures, there is sometimes a tendency to become casual. This is typically seen in the use of check lists. Items are ticked off without being properly checked. It is at this stage that accidents may happen.

\section{REFERENCES}

[1] Talbot, Julian. "What's right with risk matrices?". Jakeman Business Solutions

[2] He Zhi. "Risk Management for overseas construction projects," International Journal of Project Management[J].1995,13,231-237. (In Chinese).

[3] Du Duanfu, "integrated integration method in risk management of major engineering projects [J]," China's management science.1996(12):24-38. (In Chinese).

[4] Engert P A, and Lansdowne Z F, "Risk matrix user's guide[R]," Bed ford:The MITRE Corporation, 1999:18 - 39.

[5] Zhang Meng, and Lin Benhai, "Subway construction project risk assessment method and its application [J],"Inspection and cost of construction supervision. 2012, 5 (2). (In Chinese).

[6] Paul D. Thompson, "The Cardiovascular Risks of Diving," RecReational Diving Fatalities WoRkshop pRoceeDings, April 8-10, 2010. 